\title{
Evolution of redback radio pulsars in globular clusters
}

\author{
O. G. Benvenuto ${ }^{1,2}$, M. A. De Vito ${ }^{1,2}$, and J. E. Horvath ${ }^{3}$ \\ 1 Facultad de Ciencias Astronómicas y Geofísicas, Universidad Nacional de La Plata, Paseo del Bosque S/N (B1900FWA), \\ La Plata, Argentina \\ e-mail: adevito@fcaglp.unlp.edu.ar \\ 2 Instituto de Astrofísica de La Plata (IALP), CCT-CONICET-UNLP. Paseo del Bosque S/N (B1900FWA), La Plata, Argentina \\ 3 Instituto de Astronomia, Geofísica e Ciências Atmosféricas, Universidade de São Paulo, R. do Matão 1226, \\ 05508-090 Cidade Universitária, São Paulo SP, Brazil
}

Received 11 April 2016 / Accepted 1 November 2016

\begin{abstract}
Context. We study the evolution of close binary systems composed of a normal, intermediate mass star and a neutron star considering a chemical composition typical of that present in globular clusters $(Z=0.001)$.

Aims. We look for similarities and differences with respect to solar composition donor stars, which we have extensively studied in the past. As a definite example, we perform an application on one of the redbacks located in a globular cluster.

Methods. We performed a detailed grid of models in order to find systems that represent the so-called redback binary radio pulsar systems with donor star masses between 0.6 and 2.0 solar masses and orbital periods in the range $0.2-0.9 \mathrm{~d}$.

Results. We find that the evolution of these binary systems is rather similar to those corresponding to solar composition objects, allowing us to account for the occurrence of redbacks in globular clusters, as the main physical ingredient is the irradiation feedback. Redback systems are in the quasi-RLOF state, that is, almost filling their corresponding Roche lobe. During the irradiation cycle the system alternates between semi-detached and detached states. While detached the system appears as a binary millisecond pulsar, called a redback. Circumstellar material, as seen in redbacks, is left behind after the previous semi-detached phase.

Conclusions. The evolution of binary radio pulsar systems considering irradiation successfully accounts for, and provides a way for, the occurrence of redback pulsars in low-metallicity environments such as globular clusters. This is the case despite possible effects of the low metal content of the donor star that could drive systems away from redback configuration.
\end{abstract}

Key words. pulsars: general - stars: evolution - binaries: close

\section{Introduction}

Redback pulsars represent a recently identified and fast growing family of binary, eclipsing radio pulsars whose companion is a low-mass star $0.1 \lesssim M_{2} / M_{\odot} \lesssim 0.7$ (being $M_{2}$ the mass of the companion) on almost circular orbits with periods $0.1 \lesssim P_{\text {orb }} / d \lesssim 1.0$. Black widows form another family of pulsars with orbital periods in the same range but with companion stars that are appreciably lighter, $M_{2} \lesssim 0.05 M_{\odot}$. Among detected redbacks, some belong to the Galactic disk population whereas others reside in globular clusters (GCs). A recent listing of these system can be found in A. Patruno's catalogue ${ }^{1}$.

At present there are different proposals concerning the formation of redback pulsars. Chen et al. (2013) studied close binary systems with evaporation, considering different efficiencies due to geometrical effects. These authors concluded that redback and black widow pulsars follow different evolutionary paths depending on the strength of evaporation, finding that black widows do not descend from redback pulsars. Smedley et al. (2015) proposed that redbacks are formed via accretion induced collapse of oxygen-neon-magnesium white dwarfs (WDs). The neutron star (NS) becomes a pulsar whose irradiation evaporates the companion star. They considered that pulsar emission inhibits further accretion, finding that the whole redback region of the plane $M_{2}-P_{\text {orb }}$, where redbacks are located, can be populated

\footnotetext{
www . apatruno . wordpress . com/about/ millisecond-pulsar-catalogue/
}

in this way. Benvenuto et al. (2014; BDVH14) considered the evolution of close binary systems (CBSs) including the effects of evaporation and irradiation feedback. These authors found that mass transfer occurs in several cycles when the system has an orbital period and donor mass in the range corresponding to redbacks. They concluded that black widows descend from redbacks. In this paper we explore this evolutionary scenario further.

Irradiation feedback (Büning \& Ritter 2004) is an important phenomenon for a detailed model of mass transfer episodes in CBSs containing a NS. When the donor star fills its Roche lobe it begins to transfer mass to the NS, which may accrete a fraction of this mass. Because matter falls down the NS gravitational well, it releases X-rays, irradiating the donor star. The irradiated part of the donor star surface becomes partially blocked to release energy emerging from its interior. This effect may make the star undergo a number of mass transfer cycles. This is in sharp contrast with the classical results of standard CBS evolution, which predict long standing, continuous mass transfer stages. This irradiation model may be not sophisticated enough to explain, for example, the light curve observed for the companion of PSR J1544+4937 (see e.g. Tang et al. 2014). Nevertheless, this model enables a comprehensive description of the irradiation feedback and its implication for BWs, which we have used in this work.

Later on, the evaporation of the donor star driven by the pulsar wind becomes important. This is strongly suggested by the observations of the famous eclipsing radio pulsar PSR 1957+20, 
Table 1. Redbacks in GCs from the Patruno catalogue.

\begin{tabular}{lccc}
\hline \hline PSR & Minimum mass $\left[M_{\odot}\right]$ & Association & {$[\mathrm{Fe} / \mathrm{H}]$} \\
\hline J0024-7204W & 0.123977 & 47Tuc & $-0.75^{\mathrm{a}}$ \\
J1701-3006B & 0.121576 & M62, NGC 6266 & $-1.15 \pm 0.02^{\mathrm{b}}$ \\
J1740-5340 $(*)$ & 0.30 & NGC 6397 & $-2.28 \pm 0.01^{\mathrm{c}}$ \\
$\mathrm{J} 1748-2021 \mathrm{D}$ & 0.121182 & NGC 6440 & $-0.56 \pm 0.02^{\mathrm{d}}$ \\
$\mathrm{J} 1748-2446 \mathrm{~A}$ & 0.087283 & Ter5 & $-0.21^{\mathrm{e}}$ \\
$\mathrm{J} 1748-2446 \mathrm{ad}$ & 0.138630 & Ter5 & -0.21 \\
$\mathrm{~J} 1748-2446 \mathrm{P}$ & 0.367339 & Ter5 & -0.21 \\
$\mathrm{~J} 1824-2452 \mathrm{H}$ & 0.169625 & M28, NGC 6626 & $-1.16 \pm 0.2^{\mathrm{f}}$ \\
J1824-2452I & 0.174484 & M28, NGC 6626 & $-1.16 \pm 0.2$ \\
J2140-2310A & 0.098838 & M30 & $-2.01 \pm 0.06^{\mathrm{g}}$ \\
\hline
\end{tabular}

Notes. The minimum estimated mass and the association are taken from ATNF data base. In the case of $(*)$, the estimated mass of the companion is that of Orosz \& van Kerkwijk (2003). The metal content for the association to which the system belongs are taken from the references quoted at the end of the table.

References. (a) Ventura et al. (2014); (b) Yong et al. (2014); (c) Lovisi et al. (2012); (d) Origlia et al. (2008); (e) Ortolani et al. (2007); (f) Smith \& Wehlau (1985); (g) Kains et al. (2013).

which is the prototype of the family of black widow pulsars (Phinney et al. 1988). This effect makes the CBS undergo strong mass and angular momentum losses, allowing models to reach orbital periods of days when the leftover donor star achieves very small mass.

We (BDVH14) presented several evolutionary tracks crossing the redback region of the $M_{2}-P_{\text {orb }}$ plane. Most of these undergo several mass transfer cycles. Typically CBSs undergo mass transfer during a small fraction of each cycle and otherwise remain detached. In the detached stage it should be possible to observe the CBS as a binary radio pulsar, while during mass transfer it may be detected as a low-mass X-ray binary system (LMXB).

During mass transfer it may also be possible to detect the radio pulsar if the accretion disk surrounding the NS suffers some instability, as is strongly suggested by some recent key observations. PSR J1023+0038 is a Galactic field redback that is detected undergoing a transition from millisecond pulsar (MSP) to LMXB (Stappers et al. 2014). PSR J1824-2452I is a redback in a GC switching between accreting millisecond X-ray pulsar and redback states (Papitto et al. 2013). During the fraction of the cycles in which the pair is detached, the donor star is very similar in size to its corresponding Roche lobe. This is in the quasi-Roche lobe overflow state (quasi-RLOF). This feature provides an interpretation (Benvenuto et al. 2015) of the recent observations of PSR J1723-2837 presented by Crawford et al. (2013) and is suggested to be important for the characterization of the redback systems.

If the radio pulsar becomes observable, thereby requiring the dissipation of the innermost part of the accretion disk, likely by some instability mechanism, the system must be surrounded by the material transferred by the donor star, which is in the RLOF state. Otherwise, if the system is in the quasi-RLOF, its neighbourhood should be populated by some material evaporated from the donor star (see Fig. 2 of Benvenuto et al. 2012, in particular inset A). For a given cycle, the evaporation rate is far weaker than the mass transfer rate at RLOF. Thus, compared to the corresponding Roche lobe, the eclipsing region of systems in the quasi-RLOF state should be smaller than that corresponding to systems in the RLOF state. There are good examples of both situations: the Galactic PSR J1723-2837 shows eclipses from material located in a region twice its Roche lobe, whereas
PSR J1824-2452H, belonging to the GC M28, shows eclipses from a region six times its Roche lobe.

All the theoretical results referred to above correspond to objects with solar composition. Because a large percentage of the detected redbacks belong to GCs (at present eight systems are located in the Galactic field and another ten are found in GCs; see Table 1), it is natural to extend our theoretical results to the case of stars with a lower metal content, which is typical of Population II. This is because for low metallicity the whole picture has to be checked. A failure to produce intermittent mass transfer and settle into the quasi-RLOF state would represent a challenge to the proposed interpretation.

The response of irradiated donor stars in binary systems is largely determined by the occurrence of an outer convective zone (OCZ). It is well known that for a given mass and evolutionary state, Population II stars are more compact than those of solar composition. It is also well known that, owing to the low metal content of the mixture, at temperatures typical of outer stellar layers, the material has a lower opacity, which in turn, directly affects the behaviour of the OCZ. Furthermore, as stated above, many redbacks belong to GCs. In this context, a detailed exploration of the whole evolution of these systems is in order. We show below that the very existence of redbacks in GCs can be accounted for by irradiated models, as for solar composition objects. The remainder of this work is organized as follows: in Sect. 2 we present our numerical results. We apply them in Sect. 3 to the case of PSR J1824-2452H. Finally, in Sect. 4 we discuss the meaning of these results and give some concluding remarks.

\section{Results}

We employed our stellar binary code originally described by Benvenuto \& De Vito (2003) with the modifications cited by BDVH14, considering irradiation feedback on to the donor star without any sensible screening by the accretion disk itself (see e.g. Lasota 2001) and neglecting radio ejection effects (Ruderman et al. 1989; Burderi et al. 2001). We considered that evaporation is not relevant for the evolutionary stages we are interested in here. In addition, in the computation of the stellar models, we neglected the effects of rotation. Other illuminating models, of an indirect type, have been considered in the literature (Romani \& Sanchez 2016) motivated by high-resolution 

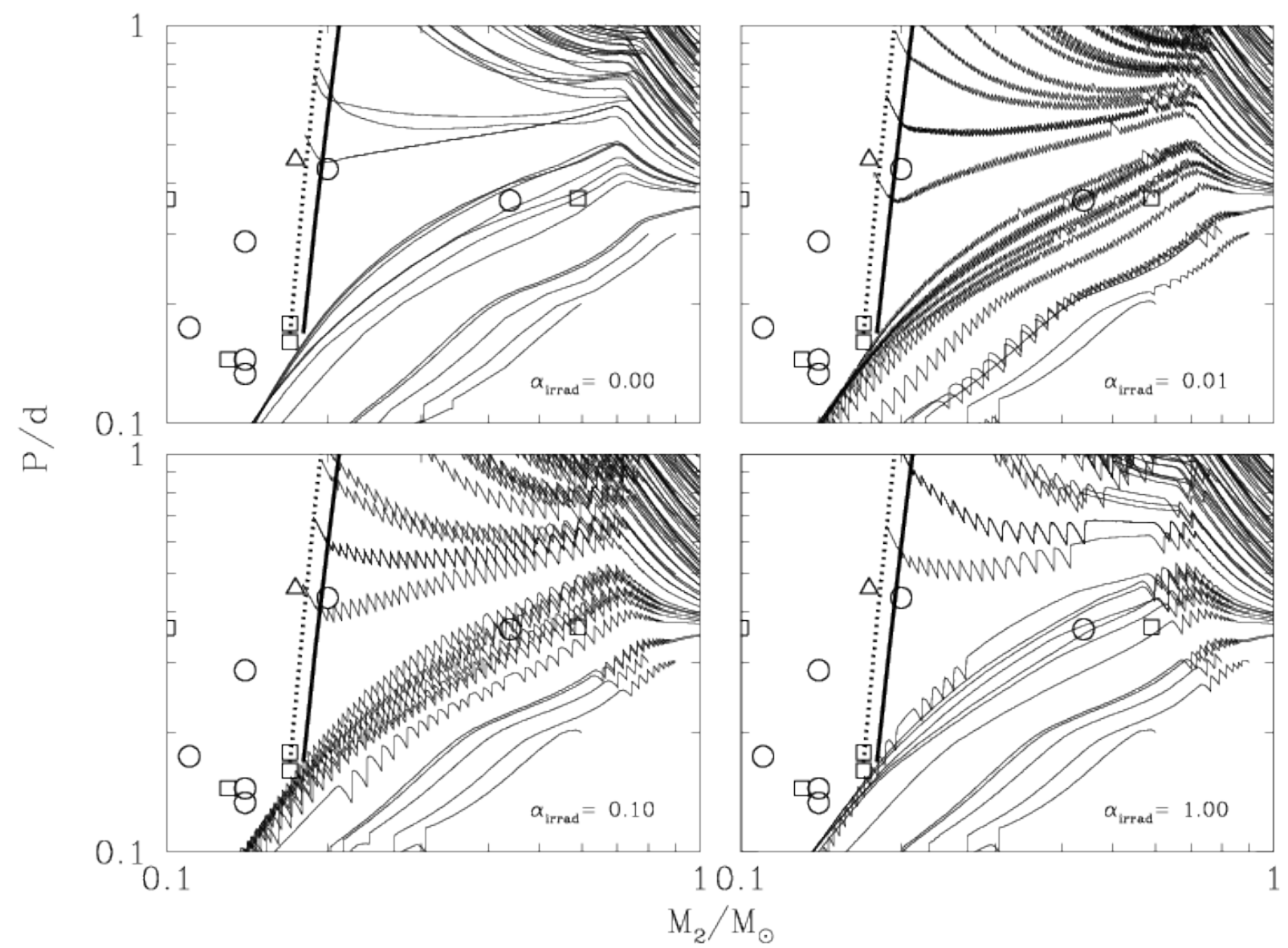

Fig. 1. Donor mass vs. orbital period for CBSs, for the case of a typical GC chemical composition $(Z=0.001)$ in the region where redbacks are located together with the corresponding observational data. Symbols denote minimum masses for radio pulsar companions. Circles and squares represent redbacks and accreting MSPs, whereas the triangle denotes the case of a system that switches between these states, all from the Patruno' catalogue $^{1}$. The thick solid (dotted) line corresponds to the mass radius relationship expected for Population II (I) composition objects given by Tauris \& Savonije (1999).

observations and these may be useful towards a compelling modelling of these systems. This possibility is not considered here and will be addressed in the future.

As BDVH14, we considered several initial masses and orbital periods considering that the NS is able to accrete a fraction $\beta$ of the mass transferred by the donor star, assuming that the absolute upper limit is given by the Eddington accretion rate, $\dot{M}_{\text {Edd }}=2 \times 10^{-8} M_{\odot} \mathrm{yr}^{-1}$. For a given set of initial conditions $\left(M_{2}, P_{\text {orb }}\right)$ we considered four different sequences with and without irradiation feedback. The accretion luminosity released by the NS is $L_{\mathrm{acc}}=\mathrm{G} M_{\mathrm{ns}} \dot{M}_{\mathrm{ns}} / R_{\mathrm{ns}}$, where $M_{\mathrm{ns}}, R_{\mathrm{ns}}$, and $\dot{M}_{\mathrm{ns}}$ are the mass, radius, and accretion rate of the NS, respectively, and $\mathrm{G}$ is the Newton gravitational constant. Assuming isotropy, the energy flux that falls onto the donor star and effectively participates in the irradiation feedback process is $F_{\text {irr }}=\alpha_{\text {irrad }} L_{\text {acc }} / 4 \pi a^{2}$. Here $a$ is the orbital radius and $\alpha_{\text {irrad }}$, considered a free parameter, is the fraction of the incident flux that effectively participates in the feedback process. We considered $\alpha_{\text {irrad }}=0.00,0.01,0.10$, and 1.00 .

Specifically, we considered an initial canonical mass of 1.4 $M_{\odot}$ for the NS, while donor star masses $M_{2}$ were considered from $0.6 M_{\odot}$ to $2.0 M_{\odot}$ with steps of $0.1 M_{\odot}$. While it is well known that there is a wide distribution for observed NS masses, the results presented in this paper are not sensitive to this mass. The initial orbital periods were set from $0.2 \mathrm{~d}$ to $0.9 \mathrm{~d}$, which is appreciably shorter than those relevant for solar composition objects because low-metallicity stars are smaller and therefore they should begin mass transfer at a closer separation. If the initial periods were longer, the systems would not evolve towards the region of the $M_{2}-P_{\text {orb }}$ plane of interest. In Table 1, we show $[\mathrm{Fe} / \mathrm{H}]$ for the $\mathrm{GCs}$ that host redback systems. The relation between the ratio $[\mathrm{Fe} / \mathrm{H}]$ and the metal content $Z$ is given by $\log _{10} Z=[\mathrm{Fe} / \mathrm{H}]-1.7$. We performed a fitting to $[\mathrm{Fe} / \mathrm{H}]$ and $Z$ taken from www.galev.org (Kotulla et al. 2009). Therefore, for the GCs listed in Table $1, Z$ lies between 0.0001 and 0.012 . In our calculations, we used a fixed metallicity of $Z=0.001$ that is representative of GCs. We do not aim in this work to perform a detailed fitting of a particular redback system in one of these GCs, but rather to explain their existence in general. We evolved these CBSs throughout the redback region.

The main results of this work are presented in Figs. 1-3. Figure 1 presents the donor mass versus orbital period relationship corresponding to the four considered cases of irradiation feedback, parametrized by $\alpha_{\text {irrad}}$. We included the data taken from the Patruno catalogue ${ }^{1}$, corresponding to redbacks, accreting X-ray MSPs, and the system PSR J1824-2452I that switches between these states, which are all observed in GCs. The observations provide minimum masses for radio pulsar companions.

If irradiation feedback is neglected $\left(\alpha_{\text {irrad }}=0.00\right)$ the systems undergo continuous RLOF. On the other hand, if irradiation feedback is allowed to operate (from $\alpha_{\text {irrad }} \gtrsim 0.01 \mathrm{on}^{2}$ ), we

2 Here we do not try to determine the minimum critical value of $\alpha_{\text {irrad }}$ for cycles to occur. 


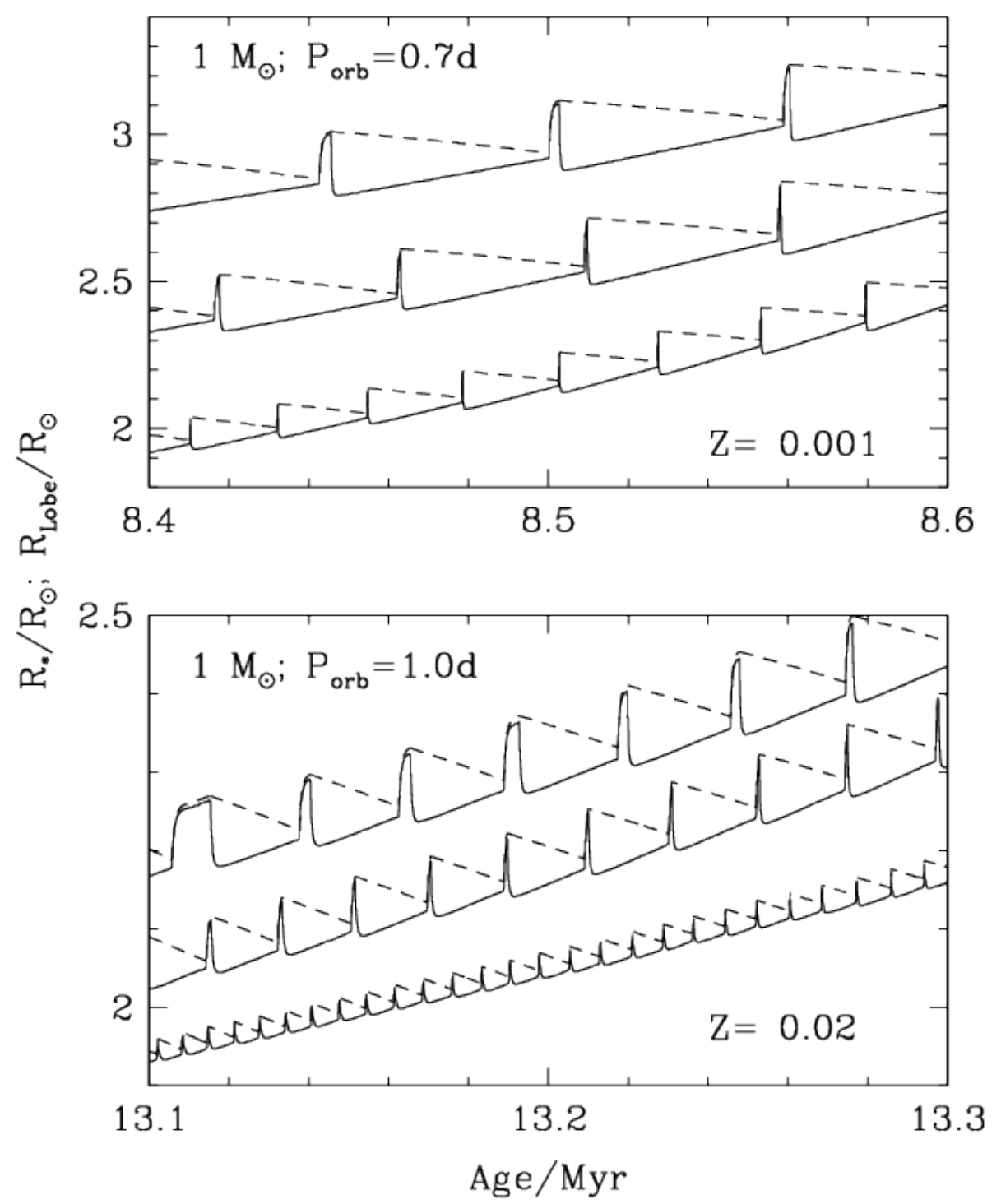

Fig. 2. Evolution of the stellar radius (solid lines) and the corresponding equivalent Roche lobe radius (dashed lines) for models with $Z=0.020$ and $Z=0.001$ (donor mass and orbital periods are indicated in each panel). For the case of $Z=0.001$ radii corresponding to $\alpha_{\text {irrad }}=0.10(1.00)$ have been subject to a vertical offset of $0.05(0.20)$. For models with $Z=0.020$ the offset was 0.5 and 1.0 , respectively.

find that the evolutionary tracks cover the same region of the $M_{2}-P_{\text {orb }}$ plane, but now undergo mass transfer cycles just as in the case of solar composition objects. The occurrence of these cycles make our models plausible to account for the detected redbacks belonging to GCs. However, these cycles do not reproduce the transitions from the state of LMXB to redback, and conversely, that have been recently observed (PSR J1023+0038, PSR J1825-2452I and XSS J12270-4859). These would respond to dynamic processes associated with the details of the accretion disk, with short timescales (of the order of months - years).

In Fig. 2 we compare the cyclic behaviour of a CBS with solar composition $\left(M_{2}=1 M_{\odot}, P_{\text {orb }}=1 \mathrm{~d}\right)$ to the case of another CBS with $Z=0.001\left(M_{2}=1 M_{\odot}, P_{\text {orb }}=0.7 \mathrm{~d}\right)$. The $P_{\text {orb }}$ for the CBS with typical GC composition was set to a lower value. This has been carried out to obtain comparable behaviours because donors with GC composition are smaller so they should be closer to the NS. In Fig. 2 we show the three $\alpha_{\text {irrad }}>0.00$ considered in this paper, finding that cycles are in general longer, therefore the total number of cycles is fewer compared to the case of solar composition models. Again, analogous to the solar composition calculations, the cycles are longer when the irradiation becomes stronger (larger $\alpha_{\text {irrad }}$ ).

These results support the idea that GC redback evolution is indeed globally similar to that of solar composition objects. In particular, redback companions should be either in RLOF or quasi-RLOF conditions. This is in agreement with the fact that donor stars nearly fill their corresponding Roche lobe as discussed by Roberts (2013). Moreover, some GC redbacks should evolve to become black widows, while others should become low-mass helium WDs (see BDVH14).

\section{Application to a specific case}

There are various redback systems in GCs with optically detected companions. The companion of PSR J1740-5340 in NGC 6397 is a bright and tidally deformed star. The luminosity and colours for this object are incompatible with a WD star (Ferraro et al. 2001). PSR J1701-3006B is in the GC NGC 6266. Its companion has been detected by Cocozza et al. (2008). Similar to the companion of PSR J1740-5340, it has a luminosity that is comparable with the turnoff of the GC but significantly 
O. G. Benvenuto et al.: Evolution of redback radio pulsars in globular clusters
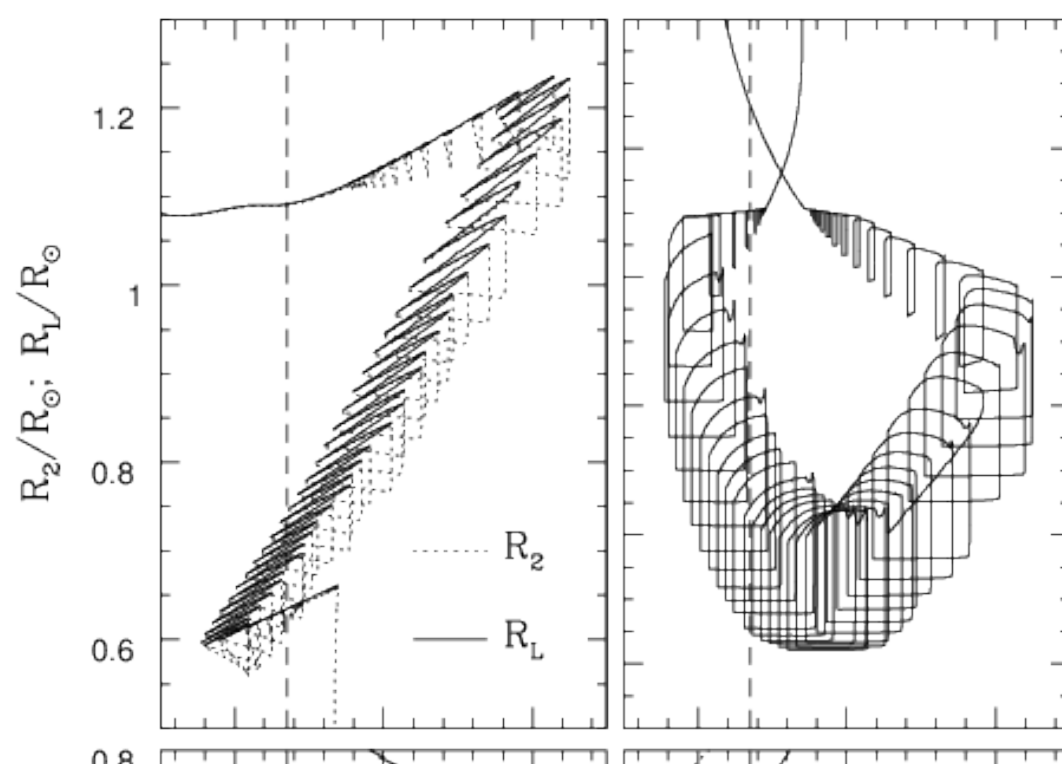

3.84
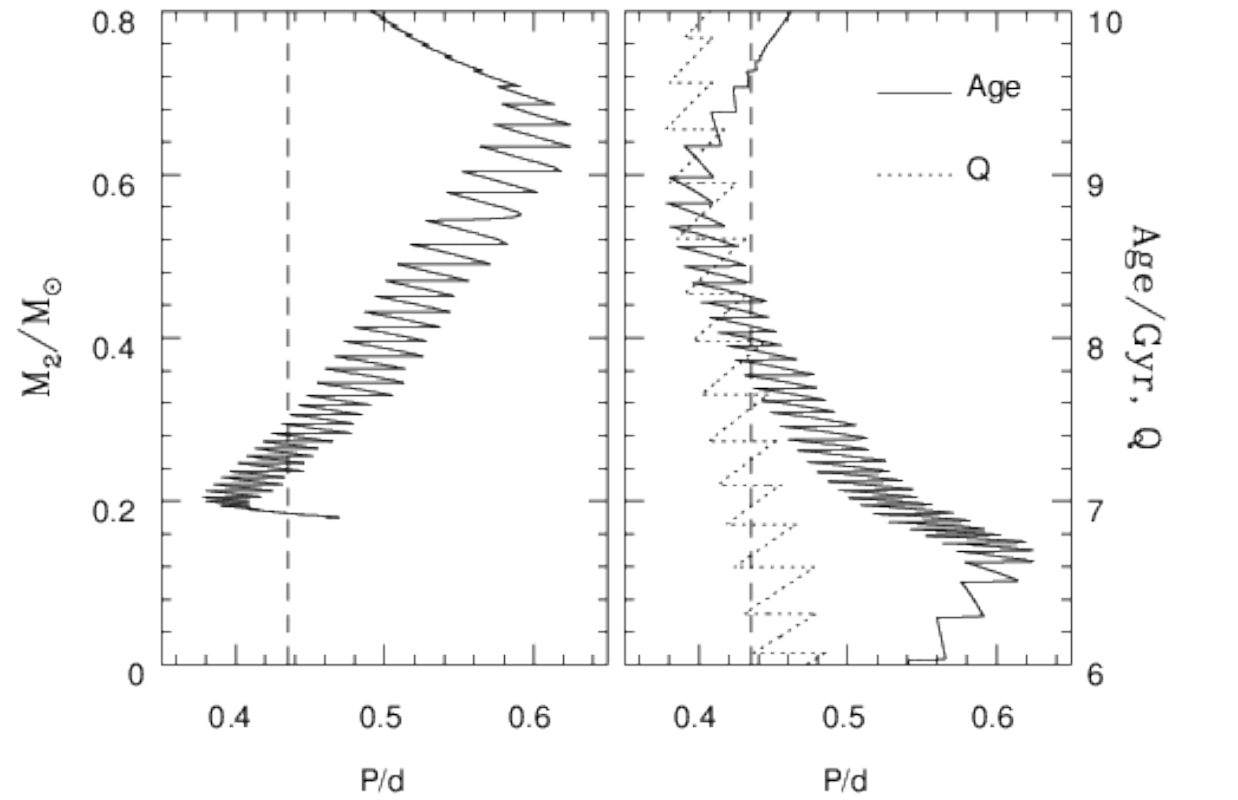

Fig. 3. Evolution of a CBS with $Z=0.001$, initial masses $M_{2}=1.30 M_{\odot}$ and $M_{\text {ns }}=1.40 M_{\odot}, P_{\text {orb }}=0.35 \mathrm{~d}, \beta=0.50$, and $\alpha_{\text {irrad }}=0.10$. All quantities are shown as a function of $P_{\text {orb }}$. Left upper panel shows the radius of the star and corresponding Roche lobe (dotted and solid lines, respectively); right upper panel depicts the effective temperature; left lower panel indicates the donor mass and the right lower panel shows the age and mass ratio (solid and dotted lines, respectively). The orbital period of PSR J1824-2452H system, $P_{\text {orb }}=0.435 \mathrm{~d}$, is shown with a vertical dashed line. The preferred $M_{2} \approx 0.2 M_{\odot}, R_{2} \approx 0.65 R_{\odot}, \log _{10}\left(T_{\text {eff }} / \mathrm{K}\right) \approx 3.78$, and $Q \approx 7$ given by Pallanca et al. (2010) are consistent with these results.

reddened. Both companion stars have high filling factors of their Roche lobe. Finally, the companion of PSR J0024-7204W was identified as a faint blue star with radio and optical observations that are compatible with a main-sequence star (Edmonds et al. 2002).

The companion of PSR J1824-2452H was identified by Pallanca et al. (2010). This system belongs to the GC M28. It has an orbital period of $P_{\text {orb }}=0.435 \mathrm{~d}$ and shows eclipses for about $20 \%$ of its period. The companion colours are $U=21.99$, $V=20.58$ and $I=19.49$. It is about $1.5 \mathrm{mag}$ fainter than the turnoff of the cluster and slightly bluer than the main-sequence. This object is too red and bright to be compatible with a WD. Based on models of Marigo et al. (2008), for isolated stars with $[\mathrm{Fe} / \mathrm{H}]=-1.27$ that are appropriate for this cluster, and assuming a colour excess $E(B-V)=0.4$ and a distance modulus
$(m-M)_{V}=14.97$ (Harris 1996), the authors estimate for the companion of PSR J1824-2452H a mass of $M_{2}=0.68 M_{\odot}$, a temperature of $6000 \mathrm{~K}$, and a radius of $R_{2}=0.64 R_{\odot}$. However, the mass function derived from radio observations $\left(f_{1}=\right.$ $\left.0.00211277 M_{\odot}\right)$, the light curve shape and the occurrence of eclipses suggest a high orbital plane inclination and, hence, an even lower mass of the companion. Assuming an inclination of $i=60^{\circ}$ (the median for the inclination angles) and a NS mass of $M_{\mathrm{ns}}=1.4 M_{\odot}$, the mass of the companion is just $M_{2} \approx 0.2 M_{\odot}$. In fact, the authors perform an iterative procedure to obtain the inclination, the mass ratio $Q=M_{\mathrm{ns}} / M_{2}$, and the Roche lobe filling factor. The best-fit model leads to $i \simeq 65^{\circ}, Q \simeq 7$ and a Roche lobe filling factor of about 1 . The size of the Roche lobe for this star is of $R_{\mathrm{L}} \approx 0.65 R_{\odot}$. This accounts for the observed luminosity of the companion of PSR J1824-2452H but seems too 
small to cause the observed radio eclipses (corresponding to an eclipsing region of about $3.3 R_{\odot}$ in size). This suggests that this material extends beyond the Roche lobe.

It is not the aim of this work to fit our models to the observed parameters of all the binary systems enumerated above. However, we applied our calculations to find a possible progenitor that accounts for the present state of one representative system, namely PSR J1824-2452H. An inspection of our models yields that the CBSs with initial masses of $M_{2}=1.30 M_{\odot}$ and $M_{\mathrm{ns}}=$ $1.40 M_{\odot}, P_{\text {orb }}=0.35 \mathrm{~d}, \beta=0.50$, and $\alpha_{\text {irrad }}=0.01,0.10$ provide a suitable evolutionary frame to characterize the PSR J1824$2452 \mathrm{H}$ system. The results corresponding to these evolutionary tracks are very similar to each other, where the main difference is the number of mass transfer cycles. Because the model with $\alpha_{\text {irrad }}=0.10$ undergoes fewer cycles, it is easier to employ for the sake of the discussion. Its main characteristics are presented in Fig. 3. In this figure, we show the radii of the donor $R_{2}$ and the Roche lobe $R_{\mathrm{L}}$ as a function $P_{\text {orb }}$ (left upper panel), effective temperature $T_{\text {eff }}$ (right upper panel), donor mass $M_{2}$ (left lower panel), and the age and mass ratio $Q$ (right lower panel). At the observed $P_{\text {orb }}$ (indicated in Fig. 3 with a vertical dashed line) we find good agreement between the observed characteristics of the donor star (Pallanca et al. 2010) and our model at an age of approximately 8 Gyr. Our theoretical age is shorter than the best fit to the main-sequence of the host cluster $(13 \mathrm{Gyr}$; Pallanca et al. 2010). There are several parameters to be selected for a CBS to study any observed object that largely affect the time the system spends to reach a given evolutionary stage; in this context the donor mass and orbital period are especially relevant. The exercise described above to characterize the evolutionary status of PSR J1824-2452H shows the suitability of the models as a tool to study the observed redbacks in GCs. However, it is not worthwhile to fit to the age of this particular system.

As stated above, PSR J1824-2452H undergoes extended eclipses corresponding to a region of about $3.3 R_{\odot}$, far larger than its associated Roche lobe and showing that the system is losing mass. Thus, this system should be in a RLOF stage and the pulsar should be visible because of accretion disk instabilities and/or radio ejection. Otherwise it would be seen as a LMXB. Still, it may be argued that CBSs with the same characteristics $\left(M_{2}, M_{\mathrm{ns}}, P_{\text {orb }}\right)$ but with $\alpha_{\text {irrad }}=0.00$ and 1.00 , which do not undergo mass transfer cycles for the same conditions corresponding to PSR J1824-2452H, may provide a suitable evolutionary frame.

\section{Discussion and conclusions}

In this work we have presented calculations of the evolution of CBSs formed by intermediate mass donor stars together with a NS for the case of low metallicity $(Z=0.0010)$, compatible with the abundances typically found in GCs. We considered standard CBS evolution and models in which irradiation feedback is included. We find that the global behaviour of CBSs is comparable to that found by BDVH14 for the case of donor stars with solar composition. As we discussed there, irradiation feedback gives rise to a cyclic mass transfer behaviour, switching from detached to semi-detached conditions, for which systems should be observable as radio pulsars and low-mass X-ray binaries, respectively. As in the case of solar composition objects, the number of mass transfer cycles undergone by a given CBS increases when $\alpha_{\text {irrad }}$ decreases. However, in the case of low-metallicity objects the number of cycles is remarkably lower than underwent by systems of high metallicity for the same $\alpha_{\text {irrad }}$.

While our models with irradiation feedback and evaporation provide a plausible scenario for the occurrence of redback pulsars, there is no reason to consider it to be the only such scenario. Indeed, other very different proposals have been presented as those of Chen et al. (2013) and Smedley et al. (2015). These works have explored possible conditions for the evolution of CBSs that is different from those we considered (very efficient evaporation and accretion induced collapse of oxygenneon-magnesium WDs). The differences between these scenarios and ours are qualitative. While we found that black widows should descend from redbacks, they claim that these families of binary pulsars are due to completely different evolutionary processes. It may be that all these ways of forming redbacks can occur in nature; whether or not this is the case is still an open problem.

Acknowledgements. We would like to thank our referee Chris Tout for his corrections and suggestions, which have helped us to improve the original version of this paper. O.G.B. is member of the Carrera del Investigador Científico of the Comisión de Investigaciones Científicas de la Provincia de Buenos Aires (CICPBA), Argentina. M.A.D.V. is member of the Carrera del Investigador Científico of the Consejo Nacional de Investigaciones Científicas y Técnicas (CONICET), Argentina. J.E.H. has been supported by Fapesp (São Paulo, Brazil) under Grant No. 2013/26258-4 and CNPq, Brazil funding agencies.

\section{References}

Benvenuto, O. G., \& De Vito, M. A. 2003, MNRAS, 342, 50 Benvenuto, O. G., De Vito, M. A., \& Horvath, J. E. 2012, ApJ, 753, L33 Benvenuto, O. G., De Vito, M. A., \& Horvath, J. E. 2014, ApJ, 786, L7 Benvenuto, O. G., De Vito, M. A., \& Horvath, J. E. 2015, ApJ, 798, 44 Büning, A., \& Ritter, H. 2004, A\&A, 423, 281

Burderi, L., Possenti, A., D’Antona, F., et al. 2001, ApJ, 560, L71

Chen, H.-L., Chen, X., Tauris, T. M., \& Han, Z. 2013, ApJ, 775, 27

Cocozza, G., Ferraro, F. R., Possenti, A., et al. 2008, ApJ, 679, L105

Crawford, F., Lyne, A. G., Stairs, I. H., et al. 2013, ApJ, 776, 20

Edmonds, P. D., Gilliland, R. L., Camilo, F., Heinke, C. O., \& Grindlay, J. E. 2002, ApJ, 579, 741

Ferraro, F. R., Possenti, A., D’Amico, N., \& Sabbi, E. 2001, ApJ, 561, L93

Harris, W. E. 1996, AJ, 112, 1487

Kains, N., Bramich, D. M., Arellano Ferro, A., et al. 2013, A\&A, 555, A36 Kotulla, R., Fritze, U., Weilbacher, P., \& Anders, P. 2009, MNRAS, 396, 462 Lasota, J.-P. 2001, New Astron. Rev., 45, 449

Lovisi, L., Mucciarelli, A., Lanzoni, B., et al. 2012, ApJ, 754, 91

Marigo, P., Girardi, L., Bressan, A., et al. 2008, A\&A, 482, 883

Origlia, L., Valenti, E., \& Rich, R. M. 2008, MNRAS, 388, 1419

Orosz, J. A., \& van Kerkwijk, M. H. 2003, A\&A, 397, 237

Ortolani, S., Barbuy, B., Bica, E., Zoccali, M., \& Renzini, A. 2007, A\&A, 470, 1043

Pallanca, C., Dalessandro, E., Ferraro, F. R., et al. 2010, ApJ, 725, 1165

Papitto, A., Ferrigno, C., Bozzo, E., et al. 2013, Nature, 501, 517

Phinney, E. S., Evans, C. R., Blandford, R. D., \& Kulkarni, S. R. 1988, Nature, 333,832

Roberts, M. S. E. 2013, in IAU Symp. 291, ed. J. van Leeuwen, 127

Romani, R. W., \& Sanchez, N. 2016, ApJ, 828, 7

Ruderman, M., Shaham, J., \& Tavani, M. 1989, ApJ, 336, 507

Smedley, S. L., Tout, C. A., Ferrario, L., \& Wickramasinghe, D. T. 2015, MNRAS, 446, 2540

Smith, H. A., \& Wehlau, A. 1985, ApJ, 298, 572

Stappers, B. W., Archibald, A. M., Hessels, J. W. T., et al. 2014, ApJ, 790, 39

Tang, S., Kaplan, D. L., Phinney, E. S., et al. 2014, ApJ, 791, L5

Tauris, T. M., \& Savonije, G. J. 1999, A\&A, 350, 928

Ventura, P., Criscienzo, M. D., D'Antona, F., et al. 2014, MNRAS, 437, 3274

Yong, D., Alves Brito, A., Da Costa, G. S., et al. 2014, MNRAS, 439, 2638 